\title{
Cepheid observations by long-baseline interferometry with FLUOR/IOTA
}

\author{
P. Kervella ${ }^{* a}$, V. Coudé du Foresto ${ }^{b}$, W. A. Traub ${ }^{c}$, M. G. Lacasse ${ }^{c}$ \\ ${ }^{a}$ European Southern Observatory, Karl-Schwartzschildstr. 2, D-85748 Garching, Germany \\ ${ }^{b}$ Observatoire de Paris, 5, place Jules Janssen, F-92195 Meudon Cedex, France \\ ${ }^{c}$ Harvard-Smithsonian Center for Astrophysics, Cambridge, Massachusetts 02138, USA
}

\begin{abstract}
We report interferometric observations of the classical galactic Cepheid $\zeta$ Gem with FLUOR (Fiber Linked Unit for Optical Recombination), installed at the IOTA (Infrared Optical Telescope Array) interferometer. Thanks to the high precision of the visibility measurements with FLUOR, it has been possible to estimate its mean uniform disk angular diameter to a relative precision of $5 \%\left(\theta_{\mathrm{ud}}=1.98 \pm 0.09 \mathrm{mas}\right)$. Variations of the angular diameter of $\zeta$ Gem were marginally detected, with an amplitude of $\Delta \theta_{\mathrm{ud}}=0.38 \pm 0.23$ mas, but further observations should allow a more precise estimation of this value. The feasibility of the observation of Cepheids with the VLTI is also evaluated. When in operation, the VLTI will allow the precise measurement of the angular diameters of a large number of Cepheids. The zero point of the period-luminosity relation could then be set with a precision of less than 0.1 mag.
\end{abstract}

Keywords: Cepheid stars, optical and infrared interferometry

\section{INTRODUCTION}

Cepheids are pulsating variable stars with a particular importance due to their period-luminosity relation. They are still today the primary distance indicators for cosmological distances. The calibration of the zero-point of the period-luminosity function is therefore of high astrophysical importance. Even the closest Cepheids are very far away. The closest one, namely $\delta$ Cephei, is at a distance of $240+/-24 \mathrm{pc}^{1}$. Observations of this star with the GI2T ${ }^{1}$ have demonstrated the capability of optical interferometry to measure its very small angular diameter (limb darkened angular diameter $\theta_{\text {ld }}=1.60 \pm 0.12$ milliarcsec). . Several Cepheid observation programs are currently also carried out at other major interferometers $\left(\mathrm{NPOI}^{3}\right.$, PTI...). This tiny angular size makes it more difficult to calibrate the zero-point of the period-luminosity function accurately. The very high resolution achievable with long-baseline interferometry allows to have access to the direct measurement of the angular diameter of Cepheids, and therefore to calibrate their distance and intrinsic luminosity ${ }^{2}$ with unprecedented precision.

\section{INSTRUMENT}

We used the IOTA interferometer ${ }^{4}$, which is located on top of Mount Hopkins, Arizona, equipped with the FLUOR ${ }^{5}$ (Fiber Linked Unit for Optical Recombination) beam combiner. It is a two telescopes interferometer with variable baselines, selectable from 5 to 38 meters. The aperture of the telescopes is $45 \mathrm{~cm}$. A third telescope has been installed recently, and will eventually enable closure phase measurements. The very small expected angular diameter of $\zeta$ Gem made the choice of the $38 \mathrm{~m}$ baseline very easy, as it is the longest available, in order to have the highest resolving power.

FLUOR is based on very thin (core diameter $6.5 \mu \mathrm{m}$ ) fluoride glass optical fibers, which are single-mode in the K-band (central wavelength $2.2 \mu \mathrm{m}$ ). The beam combiner accepts the light from the two siderostats, combines them in the fibered coupler, and produces four output signals: two interferometric signals (resulting mixed signals with antiphased fringe patterns) and two photometric signals (derived from each input beam). In this combiner, the mixing of the two electric fields is achieved by evanescent coupling between the two fiber cores, as they brought very close to each other. The simultaneous spatial filtering and photometric calibration of the interferometric signals allow to obtain very high precision visibility measurements, as the resulting fringe signals very stable.

\footnotetext{
*Correspondence: Email: pkervell@eso.org
} 


\section{3. $\zeta$ GEM AND ITS CALIBRATOR STAR HD49968}

$\zeta$ Gem is the one of the brightest Cepheids in the northern sky $(\operatorname{mag}(\mathrm{V})=3.62-4.18, \operatorname{mean} \operatorname{mag}(\mathrm{K})=1.98)$. This is also the Cepheid with the largest expected angular diameter. Baade-Wesselink method estimations ${ }^{7}$ give $\mathrm{R}_{\zeta}=69.1+5.5 /-4.8 \mathrm{R}_{\text {sun }}$ and Hipparcos measured a parallax of $2.79 \pm 0.81$ mas $^{13}$. Using these two values, $\zeta$ Gem expected angular diameter could be estimated at $1.79+0.35 /-0.42$ mas. The brightness and angular diameter of this star therefore made it an accessible target for angular diameter measurement and detection of Cepheid pulsations with FLUOR.

All the interferometric fringe visibility measurements are relative measurements between two stars. One is used as a calibrator, whose visibility $V_{\text {ref }}$ is supposed known, while the other is the scientific target of interest. The measurement of the reference star raw visibility $I_{\text {ref }}$ before and after the science star allows to measure the transfer function $T$ of the interferometer through $\mathrm{T}=I_{\text {ref }} / V_{\text {ref. }}$. The observed raw instrumental visibilities of the science target $I_{\text {science }}$ are then converted into absolute visibility measurements $V_{\text {science }}$ by $V_{\text {science }}=I_{\text {science }} / T$. The careful choice of the calibrator is very important, as any visibility variation of this star will make it impossible to evaluate properly the transfer function $T$ and will therefore contaminate the final estimate of the science target visibility.

The selected reference star, HD 49968, is a bright K5III giant (this spectral type makes it a very unlikely variable star) which is not reported variable in any catalog (SIMBAD database). The angular diameter of this star was estimated very precisely by Cohen et al. ${ }^{8}$, based on photometry at infrared wavelengths. This study gives a limb-darkened angular diameter for HD 49968 of $1.93 \pm 0.02$ mas, which can be converted in a uniform disk angular diameter of $1.87 \pm 0.02$ mas. We adopted this last value in the data reduction process to estimate the transfer function of the instrument.

\section{OBSERVATIONS AND DATA REDUCTION}

$\zeta$ Gem being a winter star, our observations were obtained in March 22-25, 1999, over four consecutive nights. $\zeta$ Gem and HD49968 were observed for sequences of about thirty minutes each alternatively. During each sequence, a few hundred interferograms were acquired, of which $50 \%$ to $95 \%$ were suitable for reduction. The fraction reduced depends on the seeing conditions, as the atmospheric piston occasionally moves the fringes out of the detection temporal window, or affects too much the fringes themselves (distortion). Table 1 gives the calibrated visibilities for $\zeta$ Gem. The data reduction procedure we used has been described in details by Coudé du Foresto et al. ${ }^{6}$. The interferometric efficiency (plotted in Figure 1) was stable within $\pm 1 \%$ over the 2 hours of observing time every night.

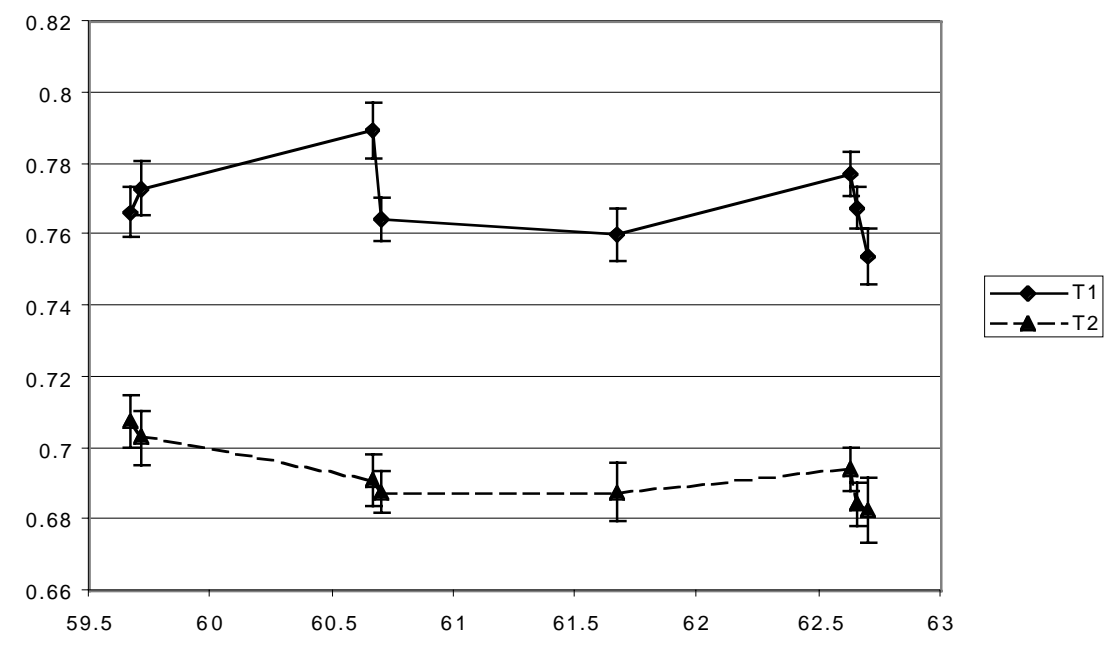

Figure 1. Transfer function of FLUOR/IOTA over the four nights of $\zeta$ Gem observations

The reference period $\mathrm{P}$ and epoch T0 for the computation of the phases are $\mathrm{P}=10.150079$ days and $\mathrm{T} 0=2444932.736$ (from Bersier et al. ${ }^{9}$ ). 
Table 1. Visibility measurements on $\zeta$ Gem

\begin{tabular}{|c|c|c|c|c|}
\hline $\begin{array}{c}\text { Julian date } \\
2451200+\ldots\end{array}$ & Phase & $\begin{array}{c}\text { Number } \\
\text { of scans }\end{array}$ & $\begin{array}{c}\text { Spatial } \\
\text { frequency } \\
\text { (cycles/arcsec) }\end{array}$ & $\begin{array}{c}\text { Calibrated } \\
\text { Visibility }\end{array}$ \\
\hline 59.69638 & 0.35000 & 131 & 83.13 & $0.9558 \pm 0.0070$ \\
\hline 60.62911 & 0.43289 & 111 & 84.00 & $0.9795 \pm 0.0103$ \\
\hline 60.68209 & 0.43811 & 122 & 83.14 & $0.9647 \pm 0.0073$ \\
\hline 61.64806 & 0.53328 & 155 & 83.51 & $0.9829 \pm 0.0098$ \\
\hline 61.68507 & 0.53693 & 167 & 83.13 & $0.9572 \pm 0.0096$ \\
\hline 62.60984 & 0.62804 & 152 & 84.37 & $0.9670 \pm 0.0079$ \\
\hline 62.63951 & 0.63096 & 156 & 83.62 & $0.9761 \pm 0.0070$ \\
\hline 62.67553 & 0.63448 & 90 & 83.15 & $0.9687 \pm 0.0103$ \\
\hline
\end{tabular}

An interesting point is the very high visibilities that were measured, in the range $95-98 \%$ : in spite of the small size of $\zeta$ Gem, visibilities were measured with a sufficient accuracy to allow precise angular diameter estimations.

\section{DATA ANALYSIS}

The goal of our study is to measure the mean angular diameter of $\zeta$ Gem accurately, and to search for the observational signature of its pulsations. As the angular diameter of this star is supposed to be variable, it is important to take into account from the beginning the information we have on these variations. In order to fit our angular diameter measurements with the most relevant model, we integrated the high precision radial velocity curve obtained on $\zeta$ Gem with the CORAVEL spectrograph ${ }^{9}$. This curve gives us the photospheric angular diameter variation curve shape, modulo the amplitude of the pulsation. We then adjusted the radius function in the visibilities space to our observational data using a classical $\chi^{2}$ minimization algorithm on two parameters : the mean angular diameter and the variation amplitude.

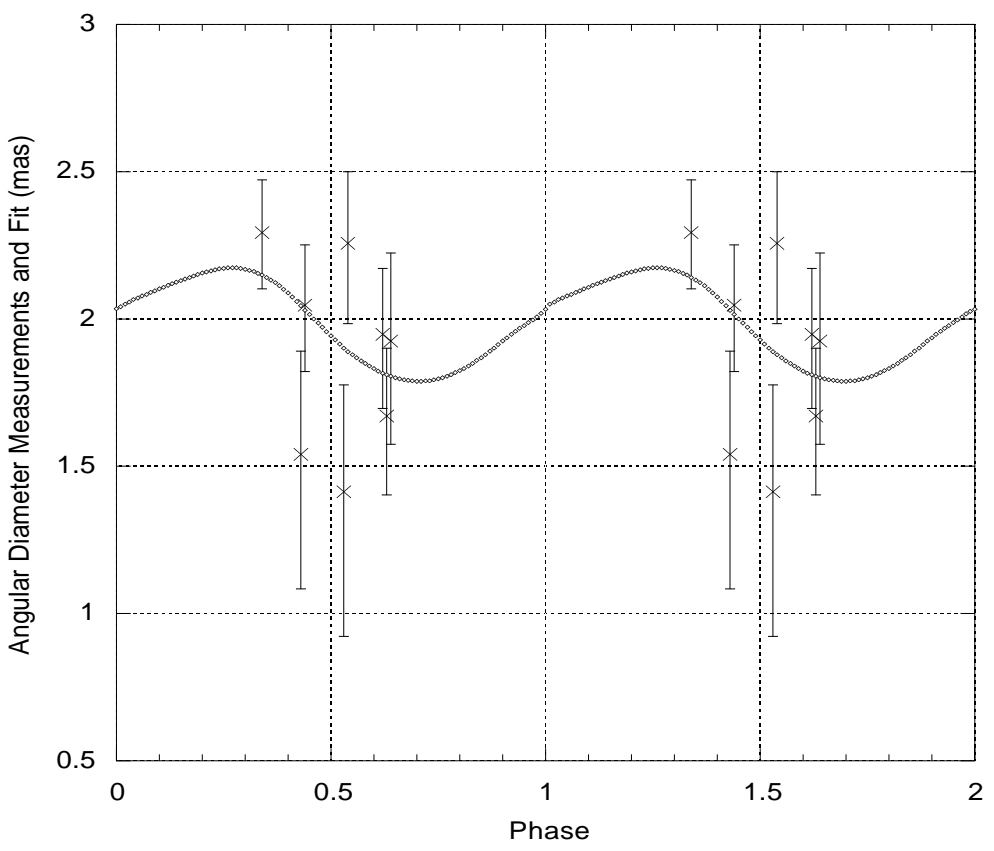

Figure 2. $\zeta$ Gem angular diameter measurements and model fit 
The uniform disk angular diameter mean value and variation amplitude were found to be $\theta_{\mathrm{ud}}=1.98 \pm 0.09$ mas and $\Delta \theta_{\mathrm{ud}}=$ $0.38 \pm 0.23$ mas.

Our angular diameter measurement is consistent with the estimation based on Hipparcos parallax and Baade-Wesselink diameter of $1.79+0.35 /-0.42$ mas. Ridgway et al. ${ }^{10}$ measured a uniform disk angular diameter of $1.81 \pm 0.31 \mathrm{mas}$ in the $\mathrm{K}$ band $(\lambda=2.17 \mu \mathrm{m})$ using the lunar occultation technique. This measurement was obtained at phase 0.596 , for which our determination is $1.83 \pm 0.09$ mas. The two independent techniques are thus in very good agreement.

\section{FUTURE CEPHEID OBSERVATIONS WITH THE VLTI}

It is expected that the VLT Interferometer ${ }^{11}$ (up to $200 \mathrm{~m}$ baseline with fringe tracking and spatial filtering) will allow precise diameter measurements on a number of Cepheids, covering a wide range of periods and distances. A preliminary list of Cepheid targets for the VLTI using the VINCI ${ }^{12}$ instrument is given in Table 2. The visibility computations have been done assuming that the longest baseline of the VLTI is used. In order to evaluate the angular diameter of these stars, the Hipparcos ${ }^{11}$ parallaxes were combined with Baade-Wesselink method diameter measurements ${ }^{14,15}$. A typical value of $10 \%$ for the pulsation amplitude has been assumed for all stars.

Table 2. The cepheids accessible to VINCI / VLTI (200 meters baseline)

\begin{tabular}{|c|c|c|c|c|c|}
\hline Name & $\begin{array}{c}\text { Mag at } \\
\text { Max(V) }\end{array}$ & Mean Mag(K) & $\begin{array}{c}\text { Mean Ang. } \\
\text { diam. (mas) }\end{array}$ & Est. Mean Visibility & $\begin{array}{c}\text { Est. Visibility } \\
\text { Variations }\end{array}$ \\
\hline l Car & 3.28 & 1.78 & 3.60 & $1.99 \%$ & $8.19 \%$ \\
\hline X Sgr & 4.20 & 2.52 & 1.65 & $47.50 \%$ & $13.12 \%$ \\
\hline$\zeta$ Gem & 3.62 & 2.12 & 2.24 & $49.50 \%$ & $12.79 \%$ \\
\hline p Dor & 3.46 & 1.96 & 1.83 & $57.94 \%$ & $11.22 \%$ \\
\hline Y Sgr & 5.25 & 3.75 & 1.44 & $58.28 \%$ & $11.14 \%$ \\
\hline K Pav & 4.40 & 2.90 & 1.95 & $58.42 \%$ & $11.12 \%$ \\
\hline$\eta$ Aql & 3.48 & 1.98 & 1.47 & $63.22 \%$ & $10.09 \%$ \\
\hline Y Oph & 5.87 & 4.37 & 1.19 & $72.28 \%$ & $7.95 \%$ \\
\hline AX Cir & 5.65 & 4.15 & 1.29 & $77.84 \%$ & $6.52 \%$ \\
\hline W Sgr & 4.29 & 2.96 & 0.86 & $83.55 \%$ & $4.95 \%$ \\
\hline S Mus & 5.89 & 4.39 & 1.15 & $85.46 \%$ & $4.41 \%$ \\
\hline AH Vel & 5.50 & 4.00 & 0.78 & $87.95 \%$ & $3.69 \%$ \\
\hline T Mon & 5.58 & 4.08 & 0.74 & $90.76 \%$ & $2.86 \%$ \\
\hline R Mus & 5.93 & 4.43 & 0.84 & $91.86 \%$ & $2.53 \%$ \\
\hline S TrA & 5.95 & 4.45 & 0.71 & $92.90 \%$ & $2.22 \%$ \\
\hline T Vul & 5.41 & 3.91 & 0.89 & $93.04 \%$ & $2.17 \%$ \\
\hline BG Cru & 5.34 & 3.84 & 0.59 & $94.50 \%$ & $1.73 \%$ \\
\hline RT Aur & 5.00 & 3.50 & 0.82 & $94.53 \%$ & $1.72 \%$ \\
\hline FF Aql & 5.18 & 3.68 & 0.48 & $96.91 \%$ & $0.98 \%$ \\
\hline S Sge & 5.24 & 3.74 & 0.40 & $97.75 \%$ & $0.71 \%$ \\
\hline MY Pup & 5.54 & 4.04 & 0.27 & $98.53 \%$ & $0.47 \%$ \\
\hline V0473 Lyr & 5.99 & 4.49 & 0.36 & $98.75 \%$ & $0.40 \%$ \\
\hline V382 Car & 3.84 & 2.34 & 0.26 & $98.93 \%$ & $0.34 \%$ \\
\hline
\end{tabular}

Based on FLUOR precision of $0.7 \%$ in about 100 interferograms, the VLTI with VINCI will measure $\zeta$ Gem diameter with a precision of \pm 0.015 mas in 30 seconds of observing time, therefore allowing the easy detection of a 0.38 mas pulsation. The measurement of Cepheids angular diameters is very important to refine the parameters of the period-luminosity relation. High precision monitoring of the angular diameter variation coupled with radial velocimetry by spectroscopy can provide direct distance measurements to these stars. The pulsation shape is essentially radial. An important problem regarding cepheids is that "we do not understand the mechanisms shaping the light curve"16: interferometry can probably help. The W Vir class of pulsating stars (Population II Cepheids) has a period-luminosity relation different from the classical cepheids law. The study of the differences between these two classes of stars will benefit a lot from the pulsations monitoring and diameter measurements by long-baseline interferometry. Possible surface features on these stars have never been studied nor detected.

The importance of Cepheid variable stars for the determination of the distances to far-away galaxies make this program a key contribution of stellar interferometry to extragalactic astronomy. Moreover, the characteristics of the visibility 
variations that are observed on Cepheids make them good intermediate steps in the search for extrasolar planets by interferometry. Their very well-defined and repeatable radial velocity curves allow the use of a synchronized detection scheme, very similar to the one that will be applied to extrasolar planetary systems.

\section{CONCLUSION}

The mean uniform disk angular diameter of the Cepheid $\zeta$ Gem has been measured using the FLUOR beam combiner and the IOTA interferometer, at a precision better than 5\%. Variations of the angular size due to pulsation of the star have been marginally detected, but further observations are planned to obtained a statistically more significant sample of the variations of this star. The new large interferometers around the world that will come into operation soon, will allow a detailed exploration of the characteristics of this class of pulsating stars.

\section{ACKNOWLEDGEMENTS}

P.K. gratefully acknowledges as a graduate student the support of the European Southern Observatory for this work.

\section{REFERENCES}

1. D. Mourard, D. Bonneau, L. Koechlin, A. Labeyrie, F. Morand, P. Stee, I. Tallon-Bosc, F. Vakili, "The mean angular diameter of $\delta$ Cephei measured by long-baseline optical interferometry”, $A \& A, \mathbf{3 1 7}$, p. 789-792, 1997.

2. D. D. Sasselov, M. Karovska, "On Cepheid diameter and distance measurement", ApJ, 432, p. 367, 1994.

3. J. T. Armstrong, R. B. Hindsley, C. A. Hummel, A. R. Hajian, D. Mozurkewich, M. E. Germain, T. E. Nordgren, "Estimating errors in stellar angular diameters: does the NPOI see the pulsation of $\delta$ Cephei?", SPIE, These Proceedings, 2000.

4. N. P. Carleton et al., "Current status of the IOTA interferometer", SPIE, 2200, p. 152-165, 1994.

5. V. Coudé du Foresto, G. Perrin, C. Ruilier, B. Mennesson, W. A. Traub, M. G. Lacasse, "FLUOR fibered instrument at the IOTA interferometer", SPIE, 3350, p. 856-863, 1998.

6. V. Coudé du Foresto, S. Ridgway, J.-M. Mariotti, "Deriving object visibilities from interferograms obtained with a fiber stellar interferometer “, A\&A Suppl. Ser., 121, p. 379-392, 1997.

7. M. Krockenberger, D. D. Sasselov, R. W. Noyes, "Radii and Distances of Cepheids. I. Method and Measurement Errors", ApJ, 479, p. 875-885, 1997.

8. M. Cohen, R. G. Walker, B. Carter, P. Hammersley, M. Kidger, K. Nuguchi "Spectral Irradiance Calibration in the Infrared. X. A Self-Consistent Radiometric All-Sky Network of Absolutely Calibrated Stellar Spectra”, AJ, 117, p. 1864-1889, 1999.

9. D. Bersier, G. Burki, M. Mayor, A. Duquennoy, "Fundamental parameters of Cepheids. II. Radial velocity data “, $A \& A S, \mathbf{1 0 8}$, p. 25-39, 1994.

10. S. T. Ridgway, G. H. Jacoby, R. R. Joyce, M. J. Siegel, D. C. Wells, “Angular diameters by the lunar occultation technique. IV - $\alpha$ Leo and the Cepheid $\zeta$ Gem”, AJ, 87, 680-684, 1982.

11. A. Glindemann, V. Coudé du Foresto, F. Delplancke, F. Derie, M. Ferrari, A. Gennai, P. Gitton, P. Kervella, B. Koehler, S. A. Lévêque, G. de Marchi, S. Menardi, A. Michel, F. Paresce, A. Richichi, M. Schoeller, A. Wallander, "VLT Interferometer: a unique instrument for high-resolution astronomy", SPIE, These Proceedings, 2000.

12. P. Kervella, V. Coudé du Foresto, A. Glindemann, R. Hofmann, "VINCI: The VLT interferometer commissioning instrument", SPIE, These Proceedings, 2000.

13. M. A. C. Perryman et al., "The Hipparcos Catalogue", A\&A, 323, 49, 1997.

14. V. Ripepi, F. Barone, L. Milano, G. Russo, " Cepheid radii and the CORS method revisited.", A\&A, 318, p. $797,1997$.

15. J. D. Fernie, "A survey of Cepheid sizes", ApJ, 282, p. 641-649, 1984.

16. A. Gautschy, H. Saio, "Stellar Pulsations Across the HR Diagram: Part 2", Ann. Rev. A\&A, 34, p. 585, 1996. 